\title{
Anderson-Bogoliubov phonon in inner crust of neutron stars: Dipole excitation in spherical Wigner-Seitz cell
}

\author{
Tsunenori Inakura ${ }^{1}$ and Masayuki Matsuo ${ }^{1}$ \\ ${ }^{1}$ Department of Physics, Faculty of Science, Niigata University, Niigata 950-2181, Japan
}

\begin{abstract}
Background: The Anderson-Bogoliubov (AB) phonon, called also the superfluid phonon, has attracted attentions since it may influence the thermal conductivity and other properties of inner crust of neutron stars. However, there are limited number of microscopic studies of the AB phonon where the presence of clusters is explicitly taken into account.

Purpose: We intend to clarify how the presence of clusters affects the AB phonon in order to obtain microscopic information relevant to the coupling between the AB phonon and the lattice phonon. Methods: The Hartree-Fock-Bogoliubov model and the quasiparticle random-phase approximation formulated in a spherical Wigner-Seitz cell are adopted to describe neutron superfluidity and associated collective excitations. We perform systematic numerical calculations for dipole excitation by varying the neutron chemical potential and the number of protons in a cell.

Results: The model predicts systematic emergence of the dipole AB phonon mode, which however exhibits strong suppression of phonon amplitude inside the cluster. We find also that the phonon amplitude around the cluster surface varies as the neutron density. At higher neutron densities the AB phonon mode exhibits behaviour similar to the pygmy dipole resonance in neutron-rich nuclei. Conclusions: The dipole $\mathrm{AB}$ phonon mode does not penetrate into the clusters. This suggests that the coupling between the AB phonon and the lattice phonon may be weak.
\end{abstract}

PACS numbers: 21.60.Jz, 21.65.Mn, 26.60.-c, 26.60.Gj, 74.20.Rp

\section{INTRODUCTION}

The inner crust of neutron stars is characteristic inhomogeneous nuclear matter that emerges in the surface region of a neutron star. In layers not very close to the neutron star core, aggregates or clusters of neutrons and protons are formed in the environment of low-density neutron gas. The clusters, which form a Coulomb lattice, resemble (but not identical to) finite nuclei while the density of neutron gas varies from $\rho_{n}=0$ to $\sim 0.1 \rho_{0}$. An important feature is that neutrons in the inner crust are believed to become superfluid at low temperatures due to the attractive nuclear force in the ${ }^{1} \mathrm{~S}_{0}$ channel.

Some of the neutron star observables are linked to the static and dynamic properties of the inner crust, characterized by the inhomogeneity and the superfluidity [13]. For example, the glitch is likely related to the pinning of superfluid vortices [4]. Cooling behaviours of young neutron stars [7, 8] and soft X-ray transients [9, 10] are governed by the heat capacity which is influenced strongly by the presence of the neutron pair gap 1113 . The quasiperiodic oscillations in giant flares are discussed in connection with the lattice vibration (lattice phonon) of clusters [14 16]. Recently another kind of dynamics, called the superfluid phonon or the Anderson-Bogoliubov (AB) phonon, has attracted attention since it is one of the lowest frequency degrees of freedom other than the lattice phonon, and it might influence thermal and mechanical properties of the inner crust $17-24$.

The superfluid phonon or the AB phonon is a collective mode of excitation which emerges generally in neutral superfluid fermions [25-27]. It is a Nambu-Goldstone mode associated with the gauge symmetry broken spontaneously by the pair condensate. Its importance as heat carrier was first discussed by Aguilera et al. [17], which points out possible new cooling mechanism effective for neutron stars under the strong magnetic field and/or low temperature. An interesting feature is that the $\mathrm{AB}$ phonon couples to the lattice phonon, and this coupling is responsible to the description of the thermal conductivity [17, 20, 21] and the quasi-periodic oscillations [20, 21]. It also might lead to formation of a new crystalline structure of the crust [23].

In the preceding works discussing the $\mathrm{AB}$ phonon in the inner crust often adopted are macroscopic approaches based on effective field theory or superfluid hydrodynamics $17-23]$. In the macroscopic models, however, the AB phonon in uniform media is assumed, and the microscopic inputs are reflected only in model parameters. A complimentary approach would be many-body theoretical descriptions based on the nucleon degrees of freedom, and such approaches may be useful to obtain microscopic information on how the $\mathrm{AB}$ phonon mode couples to the lattice phonons, and how the $\mathrm{AB}$ phonon mode evolves with the energy, the wave number, or the multipolarity. Often adopted are the selfconsistent nuclear density functional models such as the Hartree-Fock (HF) or the Hartree-Fock-Bogoliubov (HFB) methods based on all nucleon degrees of freedom. These models have been applied extensively to explore equilibrium crust configurations and to study pairing properties and the heat capacity of the matter [11 13, 28 36]. Collective excitation of inner crust matter has been studied also using the (quasiparticle) random phase approximation (RPA, QRPA) formulated on the ground of the HF/HFB models [24, 37 40]. However, the AB phonon mode in the inner crust has been investigated very little except in Ref. [37], where possible AB phonon mode is suggested 
for a low-energy quadrupole excitation, called supergiant resonance. For uniform neutron matter, a microscopic study of the AB phonon mode is performed in Ref. 24, using the density functional model and the QRPA. The contribution of the $\mathrm{AB}$ phonon mode to the heat capacity is studied. Comparison with the hydrodynamic description is discussed also.

In this paper we describe microscopically collective excitations of nuclear matter representing the inner crust of neutron stars. To describe the nucleon many-body system and its excitations, we utilize a density functional model, i.e. the Skyrme-HFB method for the equilibrium configuration and the QRPA for small amplitude modes of excitation around the equilibrium [41]. We neglect electrons for simplicity as they affects little neutron excitations such as the AB phonon mode. We adopt the Wigner-Seitz approximation so that the calculation can be performed in a single spherical Wigner-Seitz cell. In contrast to the previous works along the same line 3740], we pay special attention to the neutron pair correlation and we intend to reveal the properties of the $\mathrm{AB}$ phonon mode realized in the inner crust. As a first step of the study, we focus on the dipole excitation in the present paper since this multipolarity is responsible for the coupling to the the small amplitude displacement motion of the cluster, and hence to the lattice phonon degrees of freedom. In Sec. II, we briefly explain the model we use to describe the ground state and the excitation mode of the inner crust. In Sec. III, we discuss the static properties of the inner crusts, and the properties of the $\mathrm{AB}$ phonon mode with varying inner crust configurations. Sec. IV is devoted to the conclusion.

\section{MODELS}

We employ the HFB theory and the QRPA in order to describe the static properties and excitation modes of the inner crust. We neglect background electrons for simplicity and consider only zero temperature $T=0$. The numerical code used in the present calculation is a revised version of the HFB+QRPA code developed in Refs. 42 45] to describe isolated neutron-rich nuclei. To apply the model to the inner crust, we introduce the WignerSeitz approximation, i.e. we treat a single cell of the lattice under suitable boundary conditions. A spherical Wigner-Seitz cell is assumed, and we specify neutrons with chemical potential $\lambda_{n}(>0)$ and protons with a fixed integer number $Z$ in the spherical box. Following the standard prescriptions adopted in the $\mathrm{HF} / \mathrm{HFB}$ calculations [12, 29 36, 46], we impose the Dirichlet-Neumann boundary condition [46]; all even-parity wave functions vanish at the edge of the box, and first derivatives of odd-parity wave functions vanish at the edge of the box. Here we recapitulate the HFB+QRPA formalism briefly with emphasis on treatments relevant to the inner crust. For other details of our HFB+QRPA approach, we refer the readers to Refs. [42, 44, 45].

\section{A. Hartree-Fock-Bogoliubov theory for static properties}

The HFB equation is solved in the coordinate representation. Employing the zero-range force, the HFB equation can be written as

$$
\begin{aligned}
& \sum_{\sigma^{\prime}}\left(\begin{array}{cc}
h_{\tau}\left(\boldsymbol{r} \sigma \sigma^{\prime}\right)-\lambda_{\tau} \delta_{\sigma \sigma^{\prime}} & \tilde{h}_{\tau}\left(\boldsymbol{r} \sigma \sigma^{\prime}\right) \\
\tilde{h}_{\tau}^{*}\left(\boldsymbol{r} \sigma \sigma^{\prime}\right) & -h_{\tau}^{*}\left(\boldsymbol{r} \sigma \sigma^{\prime}\right)+\lambda_{\tau} \delta_{\sigma \sigma^{\prime}}
\end{array}\right) \phi_{i \tau}\left(\boldsymbol{r} \sigma^{\prime}\right) \\
& \quad=E \phi_{i \tau}(\boldsymbol{r} \sigma)
\end{aligned}
$$

where $\phi_{i \tau}(\boldsymbol{r} \sigma)$ is $i$-th quasiparticle wave function with isospin $\tau=n, p$ and spin $\sigma=\uparrow, \downarrow$, and $E$ is quasiparticle energy. Using the spherical symmetry, we solve the HFB equation (11) in the radial coordinate system. The HFB solution is obtained for given proton number $Z$ and neutron chemical potential $\lambda_{n}$.

The Hartree-Fock Hamiltonian $h_{\tau}$ (for the particlehole channel) is derived selfconsistently from the zerorange Skyrme effective interaction. The adopted Skyrme parameter set SLy4 [47] is the one which is adjusted to reproduce a theoretical equation of state of pure neutron matter 48] and some fundamental experiential data of isolated nuclei in wide mass range, especially neutron-rich nuclei. The center-of-mass correction $m \rightarrow m\left(1-\frac{1}{A}\right)^{-1}$ is not taken into account in the present calculation. For the pairing potential $\tilde{h}_{\tau}$ (the pairing channel), we derive it from a density-dependent delta interaction (DDDI) of the form

$$
v_{\text {pair }, \tau}\left(\boldsymbol{r}, \boldsymbol{r}^{\prime}\right)=V_{\text {pair }}\left[1-\eta\left(\frac{\rho_{\tau}(\boldsymbol{r})}{\rho_{c}}\right)^{\alpha}\right]\left(\frac{1-P_{\sigma}}{2}\right) \delta\left(\boldsymbol{r}-\boldsymbol{r}^{\prime}\right)
$$

where $\rho_{c}=0.08 \mathrm{fm}^{-3}$ and $P_{\sigma}$ is the spin-exchange operator. With this form, the pairing potential $\tilde{h}_{\tau}$ becomes a local pair potential

$$
\Delta_{\tau}(\boldsymbol{r})=V_{\text {pair }}\left[1-\eta\left(\frac{\rho_{\tau}(\boldsymbol{r})}{\rho_{c}}\right)^{\alpha}\right] \tilde{\rho}_{\tau}(\boldsymbol{r})
$$

expressed with the pair density $\tilde{\rho}_{\tau}(\boldsymbol{r})=\left\langle\Psi_{0}\right| \psi(\boldsymbol{r} \uparrow) \psi(\boldsymbol{r} \downarrow$ )$\left|\Psi_{0}\right\rangle$. The parameters are taken from Refs. [49, 50], where the overall force constant $V_{\text {pair }}=-458.4 \mathrm{MeV}$ $\mathrm{fm}^{-3}$ is determined to reproduce the ${ }^{1} \mathrm{~S}_{0}$ scattering length $a=-18.5 \mathrm{fm}$ in the free space. The remaining parameters are adjusted as $\eta=0.845$ and $\alpha=0.59$ to reproduce the neutron mater pair gap obtained from the BCS calculation using a bare nuclear force [50]. Concerning the cutoff of the quasi-particle orbits, we set $j_{\max }=(75 / 2) \hbar$ for the single-particle partial waves and we introduce the cut-off quasiparticle energy $E_{\text {cut }}=60$ $\mathrm{MeV}$ to avoid the ultraviolet divergence associated with the zero range pairing force.

\section{B. Quasiparticle random phase approximation}

The QRPA calculation is performed in order to describe excitation modes built on top of the HFB ground 
state $\Psi_{0}$. The excitation modes are classified with angular quantum numbers because of the spherical symmetry of the Wigner-Seitz cell. In the present study we focus on the dipole excitations since the dipole multipolarity is relevant to the coupling between the displacement motion of the cluster (or the lattice phonon) and the other nuclear excitations.

We utilize the linear response formalism [42, 44, 45] for the QRPA. In order to explore the pairing collectivity, the $\mathrm{AB}$ phonon mode in particular, we describe responses of the system with respect to not only the dipole moment operator $D$ but also the pair addition and removal operators $P_{\text {add }}$ and $P_{\text {rm }}$, defined by

$$
\begin{aligned}
& D=\sum_{\sigma} \int d \boldsymbol{r} r Y_{1 M}(\hat{\boldsymbol{r}}) \psi^{\dagger}(\boldsymbol{r} \sigma) \psi(\boldsymbol{r} \sigma), \\
& P_{\mathrm{add}}=\int d \boldsymbol{r} Y_{1 M}(\hat{\boldsymbol{r}}) \psi^{\dagger}(\boldsymbol{r} \downarrow) \psi^{\dagger}(\boldsymbol{r} \uparrow), \\
& P_{\mathrm{rm}}=\int d \boldsymbol{r} Y_{1 M}(\hat{\boldsymbol{r}}) \psi(\boldsymbol{r} \uparrow) \psi(\boldsymbol{r} \downarrow) .
\end{aligned}
$$

We solve the QRPA linear response equations for fluctuations of the nucleon density $\rho(\boldsymbol{r})$, the nucleon pair density $\tilde{\rho}(\boldsymbol{r})$ and its complex conjugate $\tilde{\rho}^{*}(\boldsymbol{r})$. The spectral representation is adopted for the density response function and all the quasiparticle states used in the HFB calculation are included. We calculate the strength function

$$
S(O ; E)=\sum_{M i} \delta\left(E-E_{i}\right)\left|\left\langle\Psi_{i}^{1 M}|\hat{O}| \Psi_{0}\right\rangle\right|^{2},
$$

for the operators $\hat{O}=D, P_{\text {add }}$ and $P_{\text {rm }}$. With a small imaginary constant $\epsilon$ in the energy argument, the delta function peaks in the strength functions are smeared with the Lorentzian function having the FWHM of $2 \epsilon$. We evaluate the strength $B(O)=\sum_{M}\left|\left\langle\Psi_{i}^{1 M}|\hat{O}| \Psi_{0}\right\rangle\right|^{2}$ of each excited state by integrating the strength function in an energy interval $E \in\left[E_{i}-10 \epsilon, E_{i}+10 \epsilon\right]$ around its peak energy $E_{i}$. We employ $\epsilon=10 \mathrm{keV}$. Three transition densities from the HFB ground state $\Psi_{0}$ to the $i$-th QRPA excited state $\Psi_{i}^{1 M}$

$$
\begin{aligned}
& \delta \rho_{\mathrm{ph}}(\boldsymbol{r})=\left\langle\Psi_{0}\left|\sum_{\sigma} \psi^{\dagger}(\boldsymbol{r} \sigma) \psi(\boldsymbol{r} \sigma)\right| \Psi_{i}^{1 M}\right\rangle=Y_{1 M}(\hat{\boldsymbol{r}}) \delta \rho_{\mathrm{ph}}(r), \\
& \delta \tilde{\rho}_{\mathrm{pp}}(\boldsymbol{r})=\left\langle\Psi_{0}|\psi(\boldsymbol{r} \uparrow) \psi(\boldsymbol{r} \downarrow)| \Psi_{i}^{1 M}\right\rangle=Y_{1 M}(\hat{\boldsymbol{r}}) \delta \tilde{\rho}_{\mathrm{pp}}(r), \\
& \delta \tilde{\rho}_{\mathrm{hh}}(\boldsymbol{r})=\left\langle\Psi_{0}\left|\psi^{\dagger}(\boldsymbol{r} \downarrow) \psi^{\dagger}(\boldsymbol{r} \uparrow)\right| \Psi_{i}^{1 M}\right\rangle=Y_{1 M}(\hat{\boldsymbol{r}}) \delta \tilde{\rho}_{\mathrm{hh}}(r),(6)
\end{aligned}
$$

are obtained from the corresponding fluctuating densities at the peak energy $E_{i}$. Note that all calculated spectra are discretized because of the boundary condition.

As the residual interaction to be used in the QRPA calculation, we adopt the same effective pairing interaction, Eq. (2), in the particle-particle and hole-hole channels. Concerning the residual interaction in the particlehole channel, we adopt the Landau-Migdal approximation [44, 45, 51 53] with a renormalization scheme often employed in this approximation. Namely we replace the self-consistent particle-hole interaction $v_{\mathrm{ph}}$ by the Landau-Migdal interaction $f \times v_{\text {LM }}$ derived from the Skyrme interaction and renormalized with a factor $f$. In describing isolated nuclei, this factor $f$ is fixed so that a peak corresponding to displacement motion of the nucleus emerges at zero energy. We adopt the same prescription in the present study.

\section{RESULTS AND DISCUSSION}

We have performed the HFB and QRPA calculations systematically for various configurations obtained by changing the neutron chemical potential $\lambda_{n}$ and the proton number $Z$ in order to discuss basic properties of the excitation modes of nuclear matter in the inner crust, without restricting ourselves to equilibrium configurations realized in realistic situation of the inner crust. The adopted proton numbers are $Z=20,28,40,50$, chosen to cover the range predicted in the previous $\mathrm{HFB}$ or HF calculations for the equilibrium $31-33,46]$. For the neutron chemical potential, we vary it in the range $\lambda_{n}=1-6$ $\mathrm{MeV}$, which corresponds to the density of neutron matter $\rho_{n} \approx 5 \times 10^{-4}-1 \times 10^{-2} \mathrm{fm}^{-3}=\left(3 \times 10^{-3}-7 \times 10^{-2}\right) \rho_{0}$. The box size, i.e. the radius of the Wigner-Seitz cell, is fixed to $R_{\text {box }}=20 \mathrm{fm}$ although the box size $R_{\text {box }}$ varies if we find equilibrium configurations for different layers of crust. An extension to a larger box size will be discussed in a forthcoming paper. Since dependence on the proton number is weak as shown below, we mainly discuss the case of $Z=28$ chosen as representative.

\section{A. Static properties}

Figure 1(a) shows the calculated neutron and proton densities, $\rho_{n}(r)$ and $\rho_{p}(r)$, for $Z=28$ systems with $\lambda_{n}=$ $1.0-6.0 \mathrm{MeV}$, and (b) for systems with different $Z$ with fixed $\lambda_{n}=5.0 \mathrm{MeV}$. For comparison plotted also are those for ${ }^{88} \mathrm{Ni}$ with $\lambda_{n}=-0.3 \mathrm{MeV}$, the neutron dripline isotope in the adopted HFB model. From the neutron and proton densities one can see basic features of matter composition in the inner crust: neutrons and protons aggregate together to form a cluster which resembles a nucleus while the cluster is surrounded by low-density matter of neutrons. The neutron density outside the cluster, i.e. $r \gtrsim 10 \mathrm{fm}$ in Fig. 1(a), is almost independent on $r$. The neutron density outside the cluster increases with the neutron chemical potential $\lambda_{n}$ as it should for the uniform neutron matter. Note that a slight decrease of the neutron density around $r \sim R_{\text {box }}$ is due to the specific choice of the Dirichlet-Neumann boundary condition. If we take a different boundary condition where the two parity states are treated in the opposite way, the calculated density slightly increases around $r \sim R_{\text {box }}$ in accordance with Ref. 34]. In both cases, this artificial effect of the boundary condition is small.

In contrast to the simple trend of the neutron density outside the cluster, density distributions in the re- 


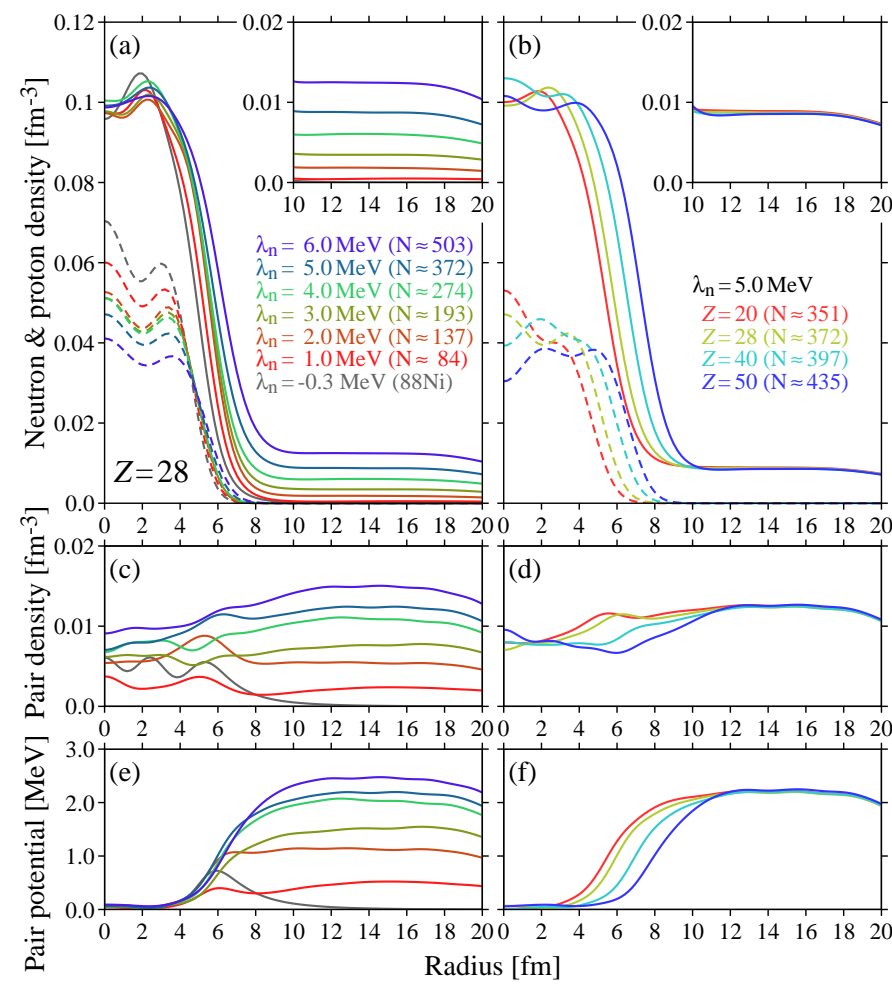

FIG. 1. (a) Calculated neutron densities (solid lines) and proton densities (dashed lines) for $Z=28$ system with $\lambda_{n}=$ $1.0-6.0 \mathrm{MeV}$ (red, brown, yellowish green, blue green, blue, and purple in order). (b) The same but for $Z=20$ (red), 28 (light green), 40 (cyan), and 50 (blue) systems with fixed $\lambda_{n}=5.0 \mathrm{MeV}$. (c)(d) Calculated neutron pair densities and (e)(f) neutron pair potentials for the same systems as (a)(b), respectively. For comparison, densities and pair potentials of drip-line nucleus ${ }^{88} \mathrm{Ni}$ are also plotted with gray line in (a) (c) (e).

gion of the cluster behave in a different way. Here we remark a few points. The increase of $\lambda_{n}$ does not increase the neutron central density of the cluster, but it induces the increase of the radius of the cluster. Evaluating the surface radius of the cluster by fitting a function $\rho_{\text {cent }} /(1+\exp [(r-R) / a])+\rho_{\text {matter }}$ to the neutron and proton densities, we have significant increase in the neutron surface radius $R_{n}=5.18,5.57,6.05 \mathrm{fm}$ for $\lambda_{n}=$ $1.0,3.0,6.0 \mathrm{MeV}$, while increase of the proton radius is smaller $\left(R_{p}=4.83,5.08,5.48 \mathrm{fm}\right.$ in the same interval). It is apparent that the neutron skin develops with increase of $\lambda_{n}$ as $R_{n}-R_{p}=0.35,0.49,0.57 \mathrm{fm}$ for $\lambda_{n}=1.0,3.0$, $6.0 \mathrm{MeV}$. Concerning dependence on the proton number $Z$ (see Fig. 1(b)), we find increase of both the neutron and proton radii with increase of $Z$ while the neutron density outside the cluster is determined solely by $\lambda_{n}$. Note that the density profile of the cluster obtained in a similar Skyrme-Hartree-Fock calculation is analyzed in Ref. [54] in terms of the equation of state of asymmetric nuclear matter.

As a measure of the superfluidity, we show the neutron

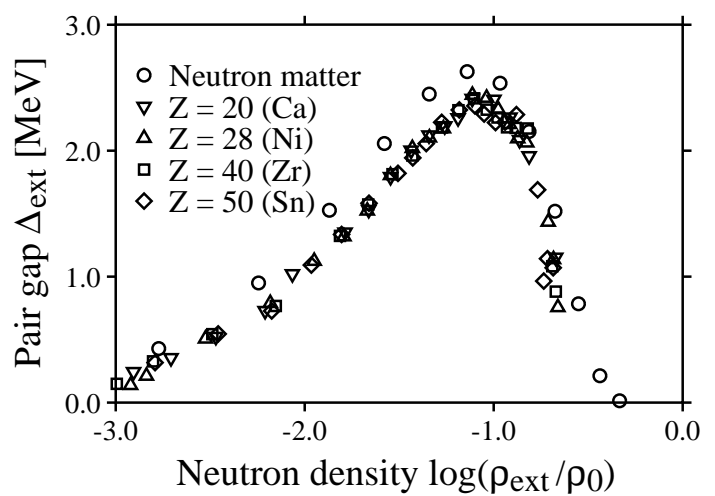

FIG. 2. Relation between neutron pair gap $\Delta_{\text {ext }}$ and neutron density $\rho_{\text {ext }}$ in the external neutron superfluid region for $Z=$ $20(\nabla), 28(\triangle), 40(\square)$, and $50(\diamond)$ systems with $\lambda_{n}$ varied up to $10 \mathrm{MeV}$. Calculated pair gap of pure neutron matter is also plotted with circles for comparison.

pair density $\tilde{\rho}_{n}(r)$ in Figs. 1(c)(d), and the neutron pair potential $\Delta(r)$ in Figs. 1(e)(f). The neutron pair density $\tilde{\rho}_{n}(r)$ exhibits a rather complex behaviour inside the surface of the cluster, but it tends to converge to a constant value in external region $r \gtrsim 12 \mathrm{fm}$ far beyond the cluster surface. It is noted that this convergence is achieved by using a large cut-off value $\left(j_{\max }=(75 / 2) \hbar\right.$ in the present calculation) of the angular momenta of the partial waves of the quasiparticle states.

For quantitative assessment of the neutron pairing properties, we evaluate the average neutron density and the average pair gap in this converged region as

$$
\rho_{\mathrm{ext}}=\frac{\int_{R_{1}}^{R_{2}} d r r^{2} \rho_{n}(r)}{\int_{R_{1}}^{R_{2}} d r r^{2}}, \quad \Delta_{\mathrm{ext}}=\frac{\int_{R_{1}}^{R_{2}} d r r^{2} \Delta_{n}(r)}{\int_{R_{1}}^{R_{2}} d r r^{2}}
$$

with $R_{1}=12 \mathrm{fm}$ and $R_{2}=18 \mathrm{fm}$. Figure 2 shows the relation between $\rho_{\text {ext }}$ and $\Delta_{\text {ext }}$, which are obtained for calcium $(Z=20)$, nickel $(Z=28)$, zirconium $(Z=40)$, and tin $(Z=50)$ systems with $\lambda_{n}$ varied up to $10 \mathrm{MeV}$. The obtained average gap $\Delta_{\text {ext }}$ is insensitive to $Z$ and displays a characteristic variation as a function of the neutron density $\rho_{\text {ext }}$ : the maximum value $\Delta_{\text {ext }} \sim 2.4$ $\mathrm{MeV}$ at density $\log \left(\rho_{\text {ext }} / \rho_{0}\right) \sim-1.0$ corresponding to $\lambda_{n} \sim 6 \mathrm{MeV}$. For comparison, we show also the neutron pair gap of uniform neutron matter calculated with the $\mathrm{HF}+\mathrm{BCS}$ methods using the same functionals (SLy4 and DDDI pairing of Eq. (2)), plotted with circles. A good agreement with the neutron matter gap is seen, and it indicates that the pairing properties of neutrons in the external region is essentially the same as those of the uniform neutron superfluid.

The pairing properties inside and in the vicinity of the cluster $(r \lesssim 12 \mathrm{fm})$ is different from those of the uniform neutron superfluid and from those of isolated nuclei as seen in Figs. 1(c)-(f). Note that the Pippard's coherence length $\xi=\hbar v_{F} /(\pi \Delta)$ in uniform neutron superfluid is estimated to be order of $5 \mathrm{fm}$ for $\rho_{n} / \rho_{0}=10^{-2}-10^{-1}$. 

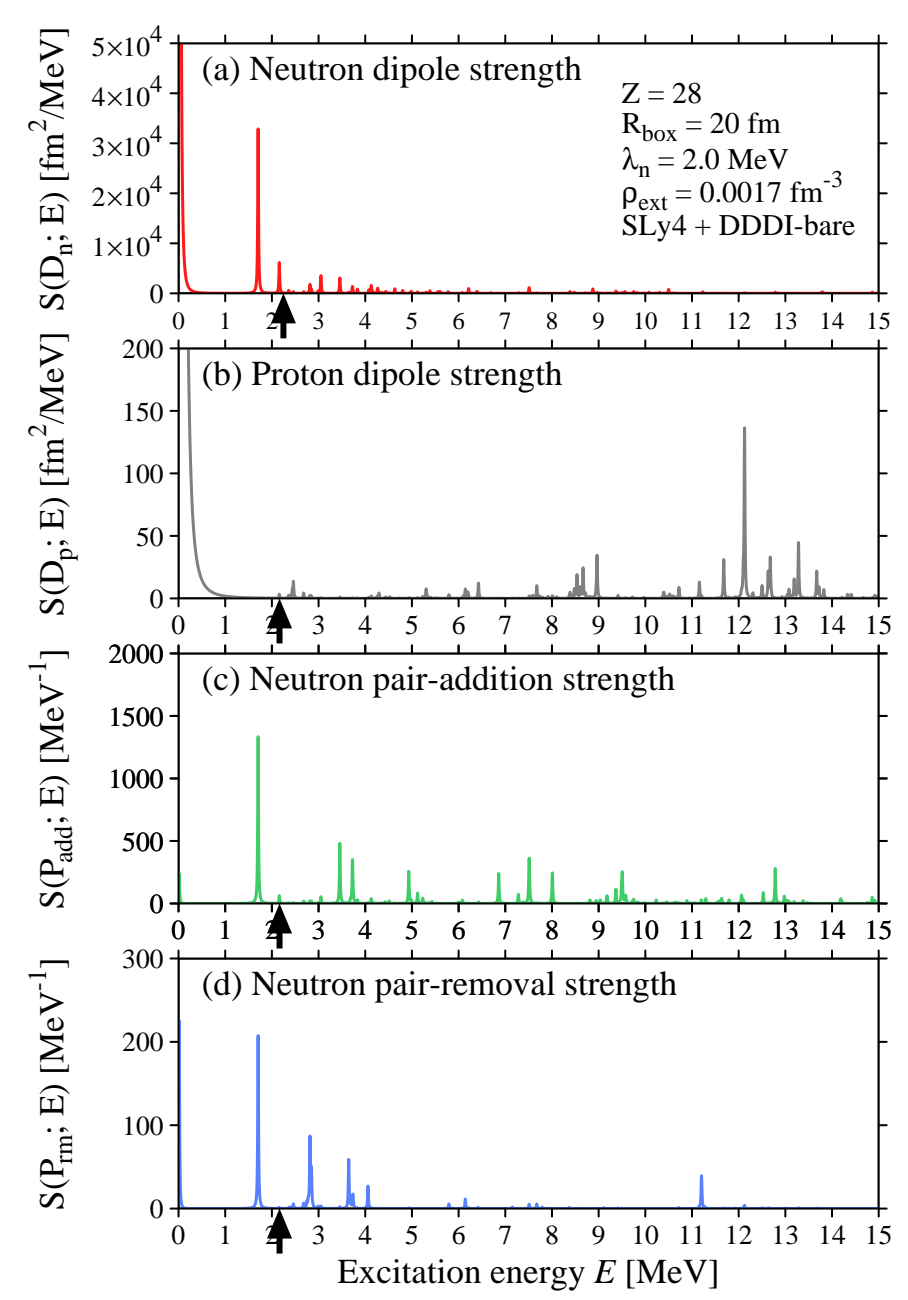

FIG. 3. (Color online) (a) Strength function $S\left(D_{n} ; E\right)$ for neutron dipole operator, (b) $S\left(D_{p} ; E\right)$ for proton dipole operator, (c) $S\left(P_{\text {add }} ; E\right)$ for neutron pair-addition operator and (d) $S\left(P_{\mathrm{rm}} ; E\right)$ for neutron pair-removal operator, calculated for $Z=28$ system with $\lambda_{n}=2.0 \mathrm{MeV}$, plotted as a function of the excitation energy $E$. Arrows indicate the threshold energy $2 \Delta_{\text {ext }}$. The smearing parameter is $\epsilon=10 \mathrm{keV}$.

The proximity effect [11, 36] is expected for the region $r \lesssim 12 \mathrm{fm}$.

\section{B. Collective dipole modes of excitations}

We shall now discuss excitation modes in the inner crust, especially those of the dipole character. Figures 3(a)-(d) show the strength functions $S(O ; E)$ for the neutron dipole moment, $O=D_{n}$, the proton dipole moment $D_{p}$, the neutron pair-addition operator $P_{\text {add }}$ and the neutron pair-removal operator $P_{\mathrm{rm}}$ for the $Z=28$ system with $\lambda_{n}=2.0 \mathrm{MeV}$.

For comparison, we show in Fig. 4 the unperturbed strength functions for the neutron dipole operator $D_{n}$

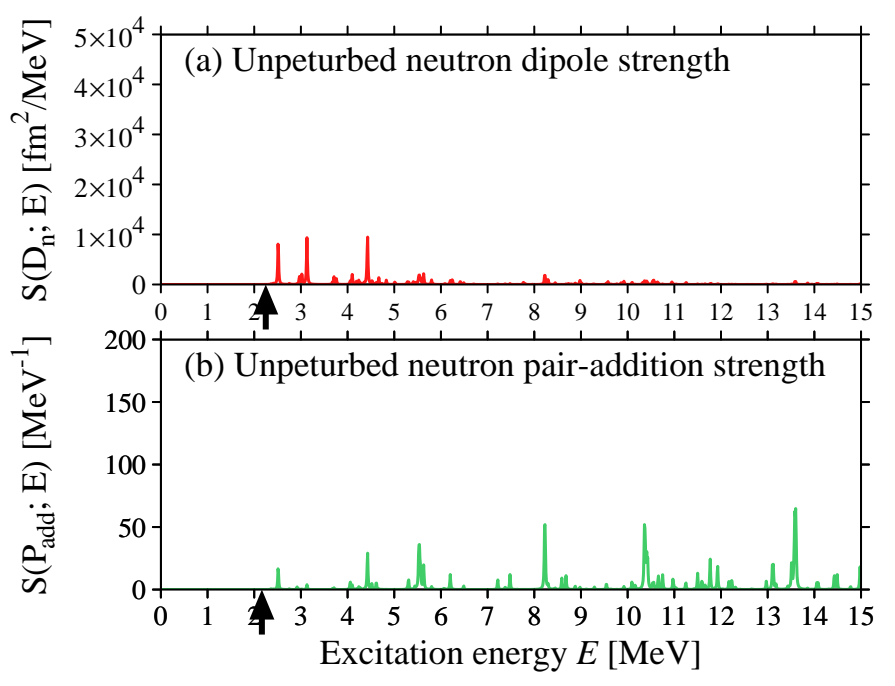

FIG. 4. (Color online) (a) Unperturbed strength function $S\left(D_{n} ; E\right)$ for neutron dipole operator and (b) $S\left(P_{\text {add }} ; E\right)$ for neutron pair-addition operator, calculated for $Z=28$ system with $\lambda_{n}=2.0 \mathrm{MeV}$. The scale of vertical axis of $S\left(P_{\text {add }} ; E\right)$ is ten times larger than that of Fig. [3(c).

and the neutron pair-addition operator $P_{\text {add }}$, i.e. those calculated for unperturbed neutron two-quasiparticle configurations. It is seen that the low-energy peaks at $E=1.71 \mathrm{MeV}$ and $E=0$ in Fig. 3 have strengths much larger than those of the unperturbed two-quasiparticle configurations. We note also that these peaks are located below twice of the neutron pair gap $2 \Delta_{\text {ext }}$ (indicated with arrows), and are well separated from densely distributed states, which would become continuum states if we take the limit of infinite $R_{\text {box }}$. These observations suggest collective nature of these low-lying peaks.

Let us examine the peak at $E=1.71 \mathrm{MeV}$. It has large strengths for the neutron dipole moment $D_{n}$, the neutron pair-addition operator $P_{\text {add }}$ and the neutron pair-removal operator $P_{\mathrm{rm}}$. This state is the largest, apart from the peak at $E=0$ (discussed below), and exhausts $40 \%, 11$ $\%$, and $25 \%$, respectively, of the total strengths (nonenergy-weighted sums integrated up to $50 \mathrm{MeV}$ excluding the zero-energy state). The neutron dipole strength $B\left(D_{n}\right)$ of this state is enhanced due to the QRPA correlation by a factor of about ten in comparison with strengths of the unperturbed two-quasi-neutron configurations. More prominent is the pair-addition and removal strengths, $B\left(P_{\text {add }}\right)$ and $B\left(P_{\mathrm{rm}}\right)$, in which drastic enhancement of order of $10^{2}$ relative to the unperturbed strengths is seen. We also note that these strengths are much larger than those of isolated nucleus by a factor of $10^{2-3}$. In contrast, the proton dipole strength of the 1.71 MeV state is negligibly small. These suggest that the $1.71 \mathrm{MeV}$ state is related to excitation of neutron superfluid rather than excitation of the cluster. In fact, this character is clearly seen in the transition densities of this state, shown in Fig. 5(a). We will discuss detailed 


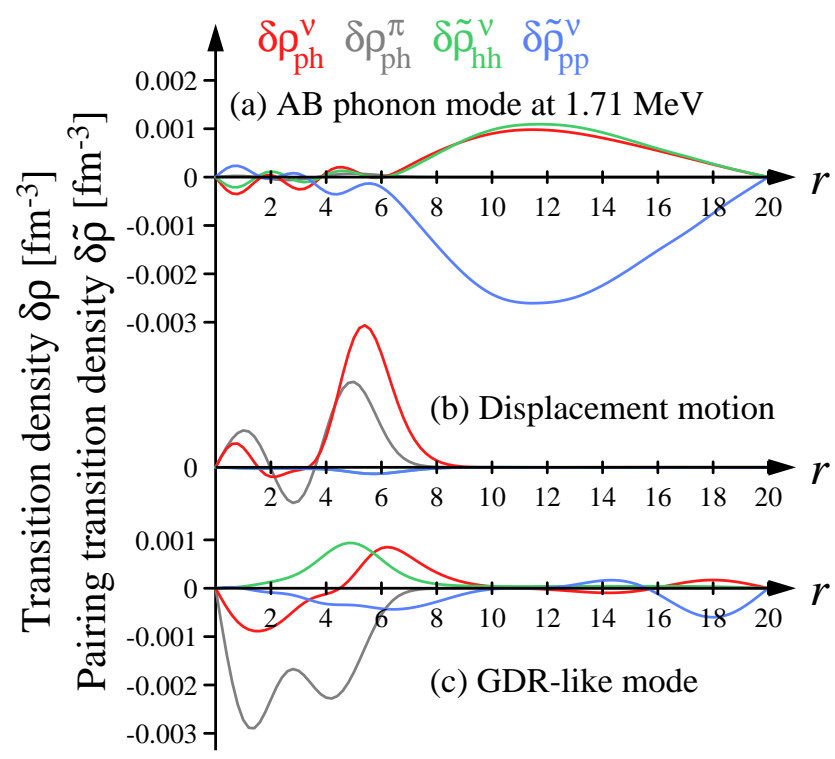

FIG. 5. (Color online) (a) Transition densities of the AB phonon mode at $E=1.71 \mathrm{MeV}$ obtained for $Z=28$ system with $\lambda_{n}=2.0 \mathrm{MeV}$. Red, gray, green, and blue lines correspond to $\delta \rho_{\mathrm{ph}}^{\nu}, \delta \rho_{\mathrm{ph}}^{\pi}, \delta \tilde{\rho}_{\mathrm{pp}}^{\nu}$, and $\delta \tilde{\rho}_{\mathrm{hh}}^{\nu}$, respectively. (b) The same but for the displacement motion of cluster at $E=0$. The scale is in arbitrary unit. (c) The same but for the GDRlike mode, calculated at $E=12.2 \mathrm{MeV}$ with employing a large smearing width $\epsilon=500 \mathrm{keV}$.

properties of this mode later.

The peak at $E=0$ is extremely intense in the neutron and proton dipole strength functions $S\left(D_{n} ; E\right)$ and $S\left(D_{p} ; E\right)$. The transition densities of this mode is shown in Fig. 5(b): Both the neutron and the proton particlehole transition densities $\delta \rho_{\mathrm{ph}}^{\nu}(r), \delta \rho_{\mathrm{ph}}^{\pi}(r)$ have large amplitudes in the surface area of the cluster, $r \approx 4-8 \mathrm{fm}$, and their shapes resemble to the derivatives $d \rho_{n}(r) / d r$ and $d \rho_{p}(r) / d r$ of the densities. All these features indicate displacement motion of the cluster as the character of this mode. The displacement motion is expected to emerge at zero energy if the HFB+QRPA calculation is fully selfconsistent and the box size is infinite. In the present calculation, we force it to be located at zero by adjusting the renormalization factor $(f=0.825$ in this case). A bunch of large proton strength in $S\left(D_{p} ; E\right)$ around 12 $\mathrm{MeV}$ is excitation similar to the giant dipole resonance (GDR) in isolated nuclei. However, this GDR-like mode exhibits some differences from the GDR. For example, the neutron transition densities have finite amplitudes in the external region, shown in Fig. 5(c), which indicates that the GDR-like mode is coupled to excitations of surrounding neutron superfluid.

\section{Anderson-Bogoliubov phonon mode}

Characteristic features of the $1.71 \mathrm{MeV}$ state are seen in the transition densities (Fig. 5(a)). The most noticeable is common sinusoidal oscillatory behaviour with long wave length seen in the the three neutron transition densities $\delta \rho_{\mathrm{ph}}^{\nu}, \delta \tilde{\rho}_{\mathrm{pp}}^{\nu}$, and $\delta \tilde{\rho}_{\mathrm{hh}}^{\nu}$ in the region $r \gtrsim 6 \mathrm{fm}$ outside the cluster. It is noticed also that the pair-addition and -removal transition densities, $\delta \tilde{\rho}_{\mathrm{pp}}^{\nu}$ and $\delta \tilde{\rho}_{\mathrm{hh}}^{\nu}$, are out of phase. It is recalled here that the $\mathrm{AB}$ phonon mode is essentially the small-amplitude oscillation of the pair gap and the pair condensate, $\Delta(\boldsymbol{r}, t) \sim \Delta e^{i \varphi(\mathbf{r}, t)}, \tilde{\rho}(\boldsymbol{r}, t) \sim$ $\tilde{\rho} e^{i \varphi(\mathbf{r}, t)}$ with the phase oscillation $\varphi(\boldsymbol{r}, t)=\epsilon e^{i \mathbf{q} \cdot \mathbf{r}-i \omega t}$. The collective motion induces fluctuating pair densities $\delta \tilde{\rho}(\boldsymbol{r}, \omega) \sim i \epsilon \tilde{\rho} e^{i \mathbf{q} \cdot \mathbf{r}}$ and $\delta \tilde{\rho}(\boldsymbol{r}, \omega)^{*} \sim-\delta \tilde{\rho}(\boldsymbol{r}, \omega)$. Also fluctuation of the particle density is induced simultaneously $\delta \rho \propto \delta \tilde{\rho} \times\left(\hbar \omega / e_{F}\right)(\rho / \tilde{\rho})$ due to the hydrodynamic character of the AB phonon mode [24, 55]. These characteristic signatures of the $\mathrm{AB}$ phonon mode are observed in the three transition densities $\delta \rho_{\mathrm{ph}}^{\nu}, \delta \tilde{\rho}_{\mathrm{pp}}^{\nu}$, and $\delta \tilde{\rho}_{\mathrm{hh}}^{\nu}$ of the 1.71 $\mathrm{MeV}$ state, especially in the region of neutron superfluid, and hence we interpret it as the AB phonon mode realized in the inner crust.

It should be noticed also that the characters as the $\mathrm{AB}$ phonon mode is seen only in the region of neutron superfluid $r \gtrsim 6 \mathrm{fm}$ outside the cluster, and behaviour of the transition densities in the cluster region $r \lesssim 6 \mathrm{fm}$ is very different from that in the external region. All the neutron transition densities here are much smaller than those outside, and wiggle with short wave lengths. If it were the $\mathrm{AB}$ phonon realized in uniform superfluid, the lowest-energy (lowest harmonics) dipole eigen mode under the present Wigner-Seitz approximation would be a standing wave having a radial profile $\propto j_{1}(q r)$ (the spherical Bessel function) with nodes at $r=0, R_{\text {box }}$. The $\mathrm{AB}$ phonon mode in the present case display approximate node at $r \approx 6.5 \mathrm{fm}$ located in the skin region of the cluster, and essentially vanishing amplitude inside the cluster. This indicates that the AB phonon mode in the present calculation does not penetrate into the cluster. The dipole AB mode appears as if it is the standing wave taking place in the region $6.5 \mathrm{fm} \lesssim r<R_{\text {box }}$.

In order to investigate the origin of the characteristic features of this excitation mode, we look into roles of the residual interaction. For this purpose we shall drop off or modify some parts of the residual interaction which enters in the QRPA calculation. Figures 6(a)(b) show 1$)$ the strength functions $S\left(D_{n} ; E\right)$ and $S\left(P_{\text {add }} ; E\right)$ obtained with only the residual pairing interaction (i.e. the particle-hole part of the residual interaction is neglected) plotted with green curves, and 2) those (blue curves) obtained with only the residual particle-hole interaction (the residual pairing interaction is neglected instead). They are compared with the full QRPA result (red curves), the same as shown in Fig. 3(a). It is seen that the calculation using only the residual pairing interaction gives the result which is similar to the full QRPA calculation with small changes in the excitation energy 

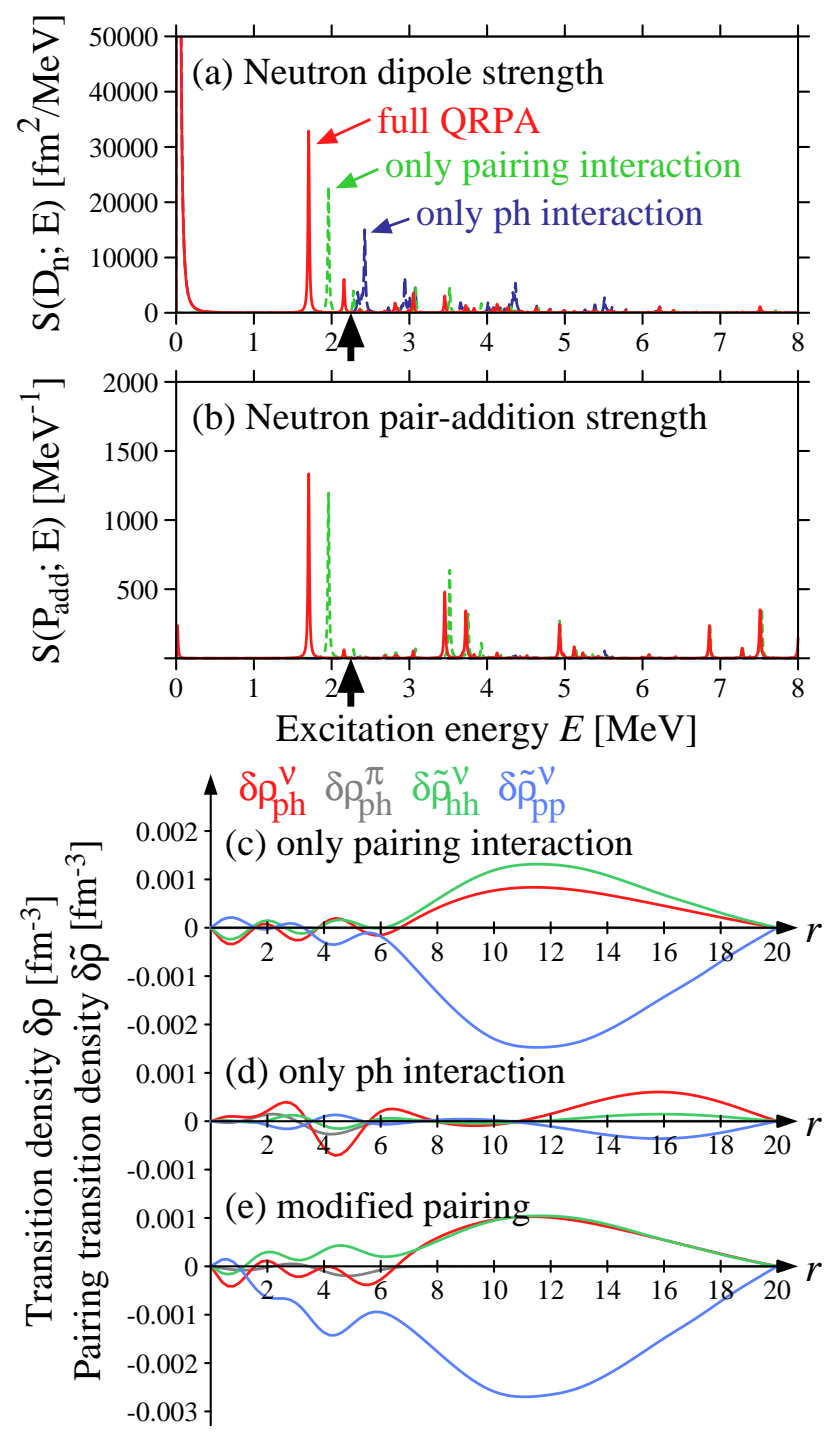

FIG. 6. (Color online) Uppers: (a) Strength function $S\left(D_{n} ; E\right)$ and (b) $S\left(P_{\text {add }} ; E\right)$ calculated with full QRPA residual interaction (red), only residual pairing interaction (green dashed), and only residual particle-hole interpretation (blue long-dashed). The system is for $Z=28$ and $\lambda_{n}=2.0 \mathrm{MeV}$. Lowers: Transition densities of the largest peak in calculations with (c) only residual pairing interaction, (d) only residual particle-hole interaction, and (e) modified pairing interaction. Red, gray, green, and blue lines correspond to $\delta \rho_{\mathrm{ph}}^{\nu}$, $\delta \rho_{\mathrm{ph}}^{\pi}, \delta \tilde{\rho}_{\mathrm{pp}}^{\nu}$, and $\delta \tilde{\rho}_{\mathrm{hh}}^{\nu}$, respectively. See text for details.

(increase to $E=1.96 \mathrm{MeV}$ ) and in the strengths (reduction of $10-30 \%$ ). In fact, the transition densities in this calculation, shown in Fig. [6(c), also resemble those of the full calculation (Fig. [5(a)) with small reduction of the neutron particle-hole transition density. If the residual pairing interaction is neglected (and only the particlehole interaction included), however, the result is completely different. The excitation energy is moved upward further, $2.45 \mathrm{MeV}$, and the strength $S\left(P_{\text {add }} ; E\right)$ becomes negligibly small. The feature of the long-wave-length oscillation of the neutron superfluid is completely lost as seen in the transition densities shown in Fig. 6)(d). We thus see that the characteristic feature of the $1.71 \mathrm{MeV}$ state is caused by the pairing correlation, and we confirm that it is essentially the $\mathrm{AB}$ phonon mode of the neutron superfluid

We performed also another modified QRPA calculation where the residual pairing interaction is changed to a simple density-independent contact interaction, which is produced by replacing the density $\rho_{n}(\boldsymbol{r})$ in the densitydependent factor $\left[1-\eta\left(\frac{\rho_{n}(\boldsymbol{r})}{\rho_{c}}\right)^{\alpha}\right]$ in Eq. (2) with the neutron superfluid density $\rho_{\text {ext }}$. This has stronger interaction strength inside the cluster than the original one while the interaction strength in the neutron superfluid region is unchanged. In this calculation the lowest excited state (excluding the displacement motion) appears at $E=1.62 \mathrm{MeV}$ with small difference from the original value $1.71 \mathrm{MeV}$. Influence of the modified pairing interaction is seen in the transition densities in the region $r \lesssim 6 \mathrm{fm}$, see Fig. 6(e): The neutron pair transition densities $\delta \tilde{\rho}_{\mathrm{pp}}^{\nu}(r)$ and $\delta \tilde{\rho}_{\mathrm{hh}}^{\nu}(r)$ are enhanced inside the cluster as is expected from the increased pairing interaction in this region. It is noted however that the pair transition densities $\delta \tilde{\rho}_{\mathrm{pp}}^{\nu}(r)$ and $\delta \tilde{\rho}_{\mathrm{hh}}^{\nu}(r)$ wiggle there (suggesting single-particle character), and the amplitude of the neutron particle-hole transition density $\delta \rho_{\mathrm{ph}}^{\nu}(r)$ remains small. The hydrodynamic AB phonon mode is not realized inside the cluster even with this modified pairing interaction.

We here point out that if we consider the coherence length $\xi=\hbar v_{F} /(\pi \Delta)$ of neutron pair correlation using the Fermi velocity $v_{F}$ and the pairing gap $\Delta$ appropriate for inside the cluster $\left(\hbar v_{F} \approx 60 \mathrm{MeVfm}, \Delta \approx 0.2 \mathrm{MeV}\right)$, it is estimated to be $\xi_{\text {int }} \approx 100 \mathrm{fm}$, much larger than the size $R_{\text {cluster }}$ of the cluster. In this situation the hydrodynamic feature of the $\mathrm{AB}$ phonon mode is hardly realized as finite-size effects might dominate there because of $R_{\text {cluster }}<\xi_{\text {int }}$. In contrast, the coherence length in the neutron superfluid outside the cluster is estimated to be $\xi_{\text {ext }}=4.5 \mathrm{fm}$. The wave length of the oscillation there is much larger than $\xi_{\text {ext }}$, thus the existence of hydrodynamic $\mathrm{AB}$ phonon mode is allowed there. This argument may be helpful to understand the feature that the $\mathrm{AB}$ phonon mode does not penetrate inside the cluster.

\section{Systematics of dipole AB phonon}

We shall discuss systematic behaviour of the dipole $A B$ phonon mode by varying $\lambda_{n}$ and $Z$. We also explore a possibility of higher harmonics of the $\mathrm{AB}$ phonon.

We first look into dependence on the neutron chemical potential $\lambda_{n}$. In Fig. 7 we plot the strength functions $S\left(D_{n} ; E\right), S\left(P_{\text {add }} ; E\right)$, and $S\left(P_{\mathrm{rm}} ; E\right)$ for $Z=28$ systems with $\lambda_{n}=1.0,2.0, \cdots, 6.0 \mathrm{MeV}$. It is seen in all the cases that low-energy collective states having large 
TABLE I. Neutron chemical potential $\lambda_{n}$, neutron density $\rho_{\text {ext }}$ and neutron pair gap $\Delta_{\text {ext }}$ outside the cluster, and excitation energy $E_{\mathrm{AB}}[\mathrm{MeV}]$, neutron dipole strength $B\left(D_{n}\right)$, proton dipole strength $B\left(D_{p}\right)$, neutron pair-addition strength $B\left(P_{\text {add }}\right)$, and neutron pair-removal strength $B\left(P_{\mathrm{rm}}\right)$ of the dipole AB phonon mode obtained for $Z=28$ system with $\lambda_{n}=1.0-6.0$ $\mathrm{MeV}$.

\begin{tabular}{cccccccc}
\hline \hline$\lambda_{n}[\mathrm{MeV}]$ & $\rho_{\mathrm{ext}}\left[\mathrm{fm}^{-3}\right]$ & $\Delta_{\mathrm{ext}}[\mathrm{MeV}]$ & $E_{\mathrm{AB}}[\mathrm{MeV}]$ & $B\left(D_{n}\right)\left[\mathrm{fm}^{2}\right]$ & $B\left(D_{p}\right)\left[\mathrm{fm}^{2}\right]$ & $B\left(P_{\mathrm{add}}\right)$ & $B\left(P_{\mathrm{rm}}\right)$ \\
\hline 1.0 & $4.67 \times 10^{-4}$ & 0.51 & 1.08 & $1.17 \times 10^{3}$ & - & 62.3 \\
2.0 & $1.67 \times 10^{-3}$ & 1.12 & 1.71 & $2.91 \times 10^{3}$ & - & 117.8 \\
3.0 & $3.25 \times 10^{-3}$ & 1.52 & 2.19 & $5.72 \times 10^{3}$ & 0.144 & 18.33 \\
4.0 & $5.58 \times 10^{-3}$ & 2.02 & 2.40 & $7.41 \times 10^{3}$ & 0.464 & 184.7 \\
5.0 & $8.23 \times 10^{-3}$ & 2.18 & 2.66 & $1.13 \times 10^{4}$ & 0.611 & 29.59 \\
6.0 & $1.17 \times 10^{-2}$ & 2.44 & 2.81 & $1.47 \times 10^{4}$ & 0.527 & 42.40 \\
\hline \hline
\end{tabular}

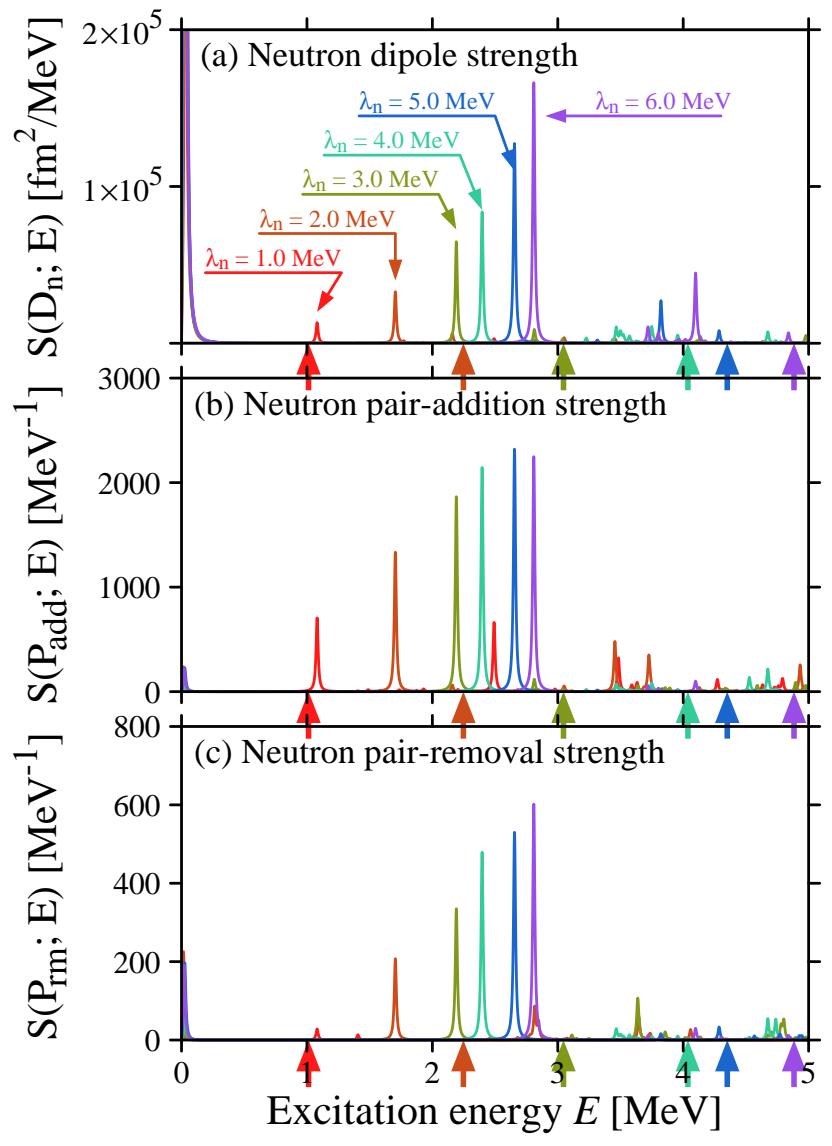

FIG. 7. (Color online) Strength functions (a) $S\left(D_{n} ; E\right)$, (b) $S\left(P_{\text {add }} ; E\right)$, and $(\mathrm{c}) S\left(P_{\mathrm{rm}} ; E\right)$ for $Z=28$ system with $\lambda_{n}=$ 1.0 MeV (red), 2.0 MeV (brown), 3.0 MeV (yellow green), 4.0 MeV (blue green), 5.0 MeV (blue), and 6.0 MeV (purple). Colored arrows indicate the threshold energy $2 \Delta_{\text {ext }}$ for corresponding $\lambda_{n}$. See text for details.

strengths emerge around $1-3 \mathrm{MeV}$ of excitation energy. The transition densities of these collective states are shown in Fig. 8 for $\lambda_{n}=1.0,3.0, \cdots, 6.0 \mathrm{MeV}$, and it is found that they all share the basic characteristics of the $\mathrm{AB}$ phonon mode found in the case $\lambda_{n}=2.0 \mathrm{MeV}$.

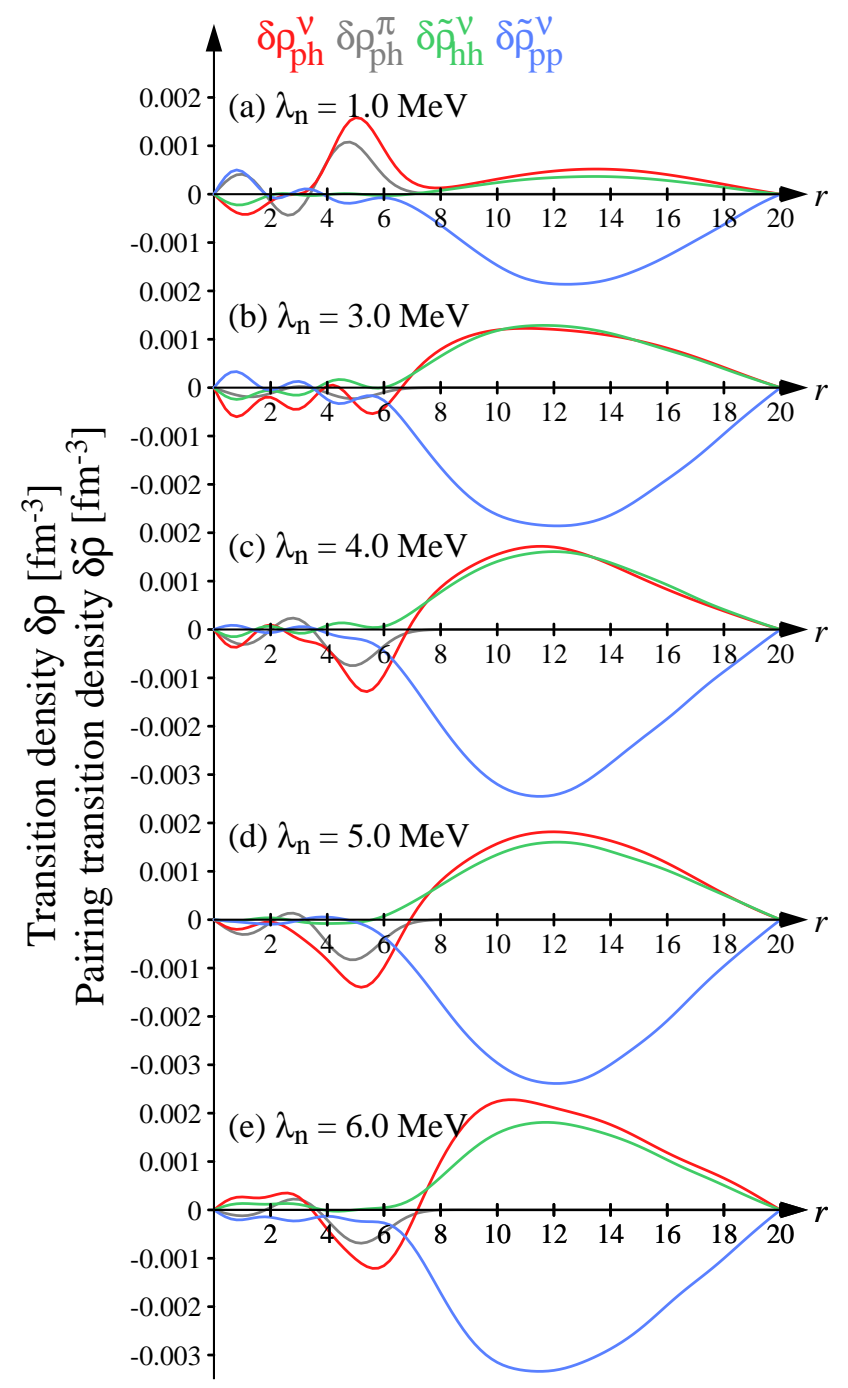

FIG. 8. (Color online) Transition densities $\delta \rho_{\mathrm{ph}}^{\nu}, \delta \rho_{\mathrm{ph}}^{\pi}, \delta \tilde{\rho}_{\mathrm{pp}}^{\nu}$, and $\delta \tilde{\rho}_{\text {hh }}^{\nu}$ of the dipole $\mathrm{AB}$ phonon mode for $Z=28$ system with $\lambda_{n}=$ (a) $1.0 \mathrm{MeV}$, (b) $3.0 \mathrm{MeV}$, (c) $4.0 \mathrm{MeV}$, (d) 5.0 $\mathrm{MeV}$ and (e) $6.0 \mathrm{MeV}$. See text for details.

Concerning the $\lambda_{n}$-dependence, the excitation energy 


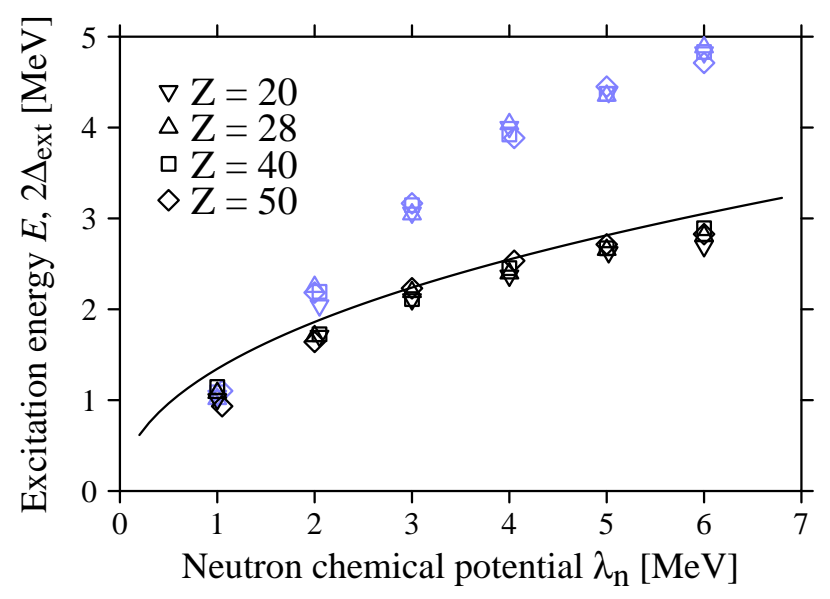

FIG. 9. (Color online) Excitation energy of the dipole AB phonon mode for $Z=20(\nabla), 28(\triangle), 40(\square)$, and $50(\diamond)$ plotted as a function of neutron chemical potential $\lambda_{n}$. Blue symbols are the threshold energy $2 \Delta_{\text {ext }}$. Hydrodynamic estimate of the excitation energy is also plotted with solid line. See text for details.

increases with increase of $\lambda_{n}$. It is seen also that the peak height of the neutron strength functions $S\left(D_{n} ; E\right)$, $S\left(P_{\text {add }} ; E\right)$ and $S\left(P_{\mathrm{rm}} ; E\right)$, and hence the transition strengths develop with increasing $\lambda_{n}$. The excitation energy and the transition strengths are listed in Table [ and the $\lambda_{n}$-dependence of the excitation energy is shown in Fig. 9 ,

The $\lambda_{n}$-dependence of the excitation energy can be assessed in a simple way. For this purpose we consider a simple hydrodynamic description of $\mathrm{AB}$ phonon, and suppose that it is modified by the presence of the cluster. Namely we assume a standing wave taking place outside the cluster. Then the phonon amplitude may be expressed as $\phi(r) \propto j_{1}(q r)+A n_{1}(q r)$ in terms of the spherical Bessel and Neumann functions together with the boundary condition $\phi(r)=0$ at the box edge $r=R_{\text {box }}=20 \mathrm{fm}$ and with the approximate node at $r \approx 7 \mathrm{fm}$ located in the surface region of cluster. This determines the wave number $q=0.265 \mathrm{fm}^{-1}$ for this standing wave. Combination of the phonon dispersion relation $\omega=c_{\mathrm{ph}} q$ and the hydrodynamic estimate of the phonon velocity $c_{\mathrm{ph}}=\sqrt{\left(\partial \lambda_{n} / \partial \rho\right) \rho / m}$ gives an estimated excitation energy $\hbar \omega_{\text {hyd }} \mathbb{1}$, which is plotted as solid curve in Fig. 9. We find reasonably good agreement between the microscopically evaluated excitation energy and this simple estimate. If we suppose a plane wave

\footnotetext{
${ }^{1}$ In this estimate, we use $\rho\left(\lambda_{n}\right)$ evaluated for uniform neutron matter with the same Skyrme functional SLy4. Note also that according to Ref. [24] the phonon dispersion $\omega=c_{\mathrm{ph}} q$ with the hydrodynamic phonon velocity $c_{\mathrm{ph}}$ may be realized for $\hbar \omega$ smaller than $2 \Delta$, but deviate as $\omega<c_{\mathrm{ph}} q$ for $\hbar \omega \gtrsim \Delta$. Therefore $\hbar \omega_{\text {hyd }}$ gives a rough upper estimate of the excitation energy.
}

phonon $\phi(r) \propto j_{1}(q r)$ in uniform neutron superfluid, i.e. without influence of the cluster, then $q=0.224 \mathrm{fm}^{-1}$ and the comparison becomes worth. All these indicate that the collective state under discussion is described in terms of the modified hydrodynamic picture in a zeroth order approximation. An exceptional example is the case of $\lambda_{n}=1.0 \mathrm{MeV}$. The collectivity may not be developed well in this case as seen in the transition densities (Fig. 8(a)) where the simple phonon behaviour is partially broken, and in the excitation energy which is slightly larger than $2 \Delta_{\text {ext }}$. Note however that we would have a well developed $\mathrm{AB}$ phonon mode even in the case of $\lambda_{n}=1 \mathrm{MeV}$ if we took such a large Wigner-Seitz cell that gives small $q$ and small excitation energy $\ll 2 \Delta_{\text {ext }}$.

In addition to the zero-th order picture discussed above, an interesting behaviour of the transition densities is seen in the cases of $\lambda_{n} \gtrsim 4.0 \mathrm{MeV}$ (Figs. 8(c)-(e)) in the region $r=3-8 \mathrm{fm}$ around the surface of the cluster. In these cases, the neutron particle-hole transition density $\delta \rho_{\mathrm{ph}}^{\nu}(r)$ exhibits a clear node at $r \approx 7 \mathrm{fm}$, and it has sizable amplitude in the region $r \approx 3-7 \mathrm{fm}$ with opposite sign to the amplitude outside $r \gtrsim 7 \mathrm{fm}$. It is seen also that the proton particle-hole amplitude $\delta \rho_{\mathrm{ph}}^{\pi}(r)$ has finite amplitude in this region with coherence to $\delta \rho_{\mathrm{ph}}^{\nu}(r)$, exhibiting some resemblance to the displacement motion of the cluster (see Fig. 5(b)). It is as if oscillation of superfluid neutrons outside the surface repels the cluster, and a core part $r \lesssim 7 \mathrm{fm}$ of the cluster receives a recoil motion in the opposite direction. However, the amplitude of the 'recoil motion' is small as deduced from the transition strength for the proton dipole operator $\sim O\left(10^{-1}\right) \mathrm{fm}^{2}$ ( Table I), which is much smaller than the single-particle values $\sim R_{\text {nucl }}^{2} \sim O\left(10^{1}\right) \mathrm{fm}^{2}$. It is interesting to note that this characteristic behaviour of the transition densities is similar to those of the pygmy dipole resonance and the low-energy dipole excitations [53] of isolated neutronrich nuclei. In the latter case, however, the oscillation in the outer area is not that of neutron superfluid, but neutron skin or extended tail of neutron density associated with weakly bound neutrons.

We shall briefly discuss dependence on the proton number $Z$ of the cluster. We have performed calculations for systems with different proton numbers $Z=$ $20,28,40,50$, corresponding to $\mathrm{Ca}, \mathrm{Ni}, \mathrm{Zr}$, and $\mathrm{Sn}$, respectively, and with various $\lambda_{n}$. We found in all the cases the $\mathrm{AB}$ phonon mode having the same character as discussed above. Examples with $\lambda_{n}=4.0 \mathrm{MeV}$ and $Z=20,28,40,50$ are shown in Figs. 10(a)(b), where are plotted the strength functions $S\left(D_{n} ; E\right)$ and $S\left(P_{\text {add }} ; E\right)$. The transition densities are shown in Figs. 10(c)-(e). It is seen in Figs. 10(a)(b) that the excitation energy varies only weakly with the change of the proton number. The transition strengths (the peak height) are also insensitive to $Z$. This is a reasonable consequence of the fact that the present $\mathrm{AB}$ phonon mode is essentially the excitation of neutron superfluid taking place only outside the cluster. We note also that the characteristic coupling between the $\mathrm{AB}$ phonon mode and the recoil motion of 

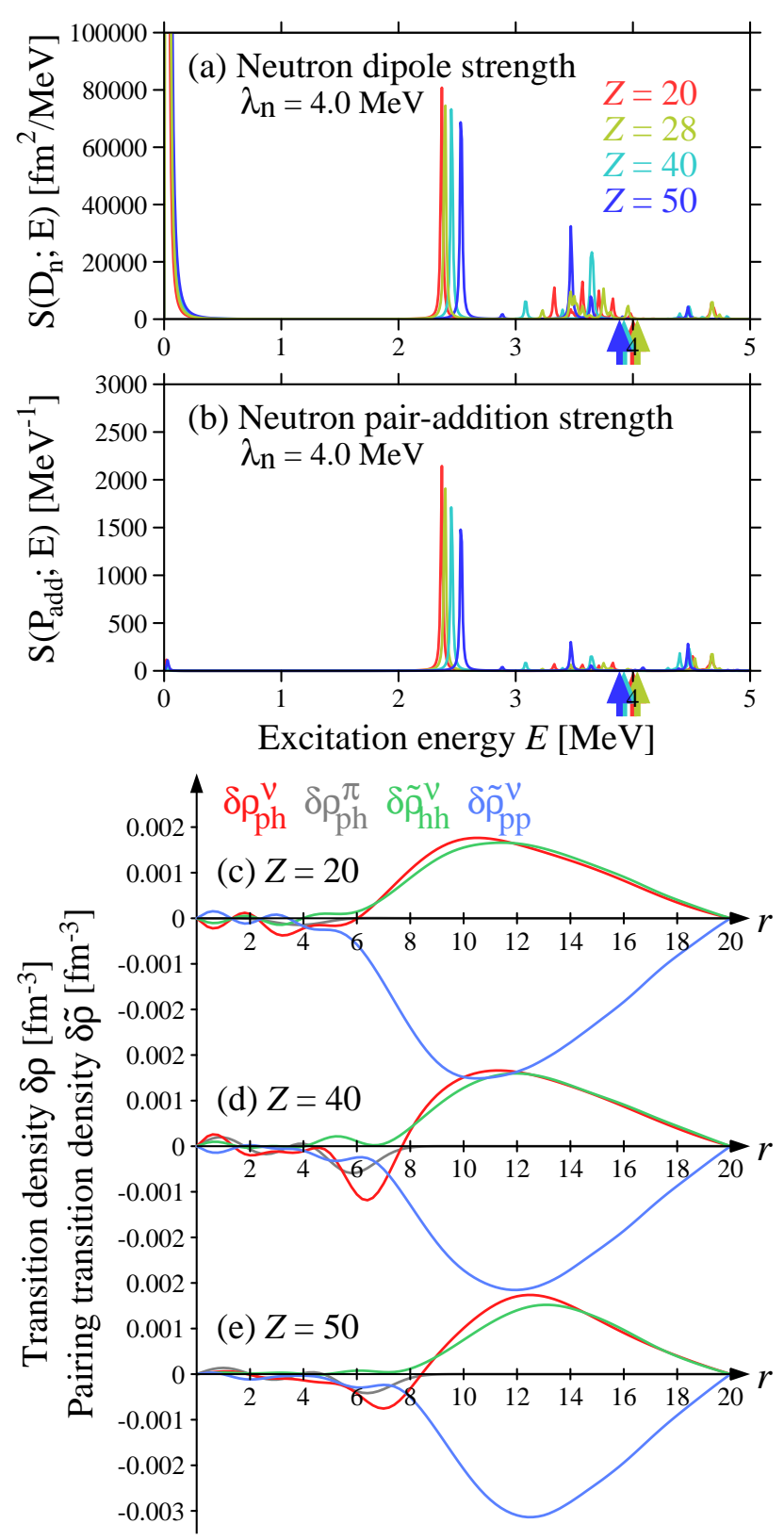

FIG. 10. (Color online) Uppers: Strength functions (a) $S\left(D_{n} ; E\right)$ and (b) $S\left(P_{\text {add }} ; E\right)$ for $Z=20$ (red), 28 (light green), 40 (cyan), and 50 (blue) systems with fixed $\lambda_{n}=$ $4.0 \mathrm{MeV}$. Colored arrows indicate the threshold energy $2 \Delta_{\text {ext }}$ for corresponding $Z$. Lowers: Transition densities $\delta \rho_{\mathrm{ph}}^{\nu}, \delta \rho_{\mathrm{ph}}^{\pi}$, $\delta \tilde{\rho}_{\mathrm{pp}}^{\nu}$, and $\delta \tilde{\rho}_{\mathrm{hh}}^{\nu}$ of the dipole $\mathrm{AB}$ phonon for (c) $Z=20$, (d) $Z=40$, and (e) $Z=50$ systems. See text for details.

the core part of cluster, demonstrated in Figs. 8(c)-(e), is seen systematically for larger values of the neutron chemical potential $\lambda_{n} \gtrsim 4-5 \mathrm{MeV}$.

Finally, we explore possibility of higher harmonics of the $\mathrm{AB}$ phonon mode, which may emerge if the collectivity of the phonon mode is sufficiently strong. In the case of $\lambda_{n}=2.0 \mathrm{MeV}$ and $Z=28$, we find that the sec-

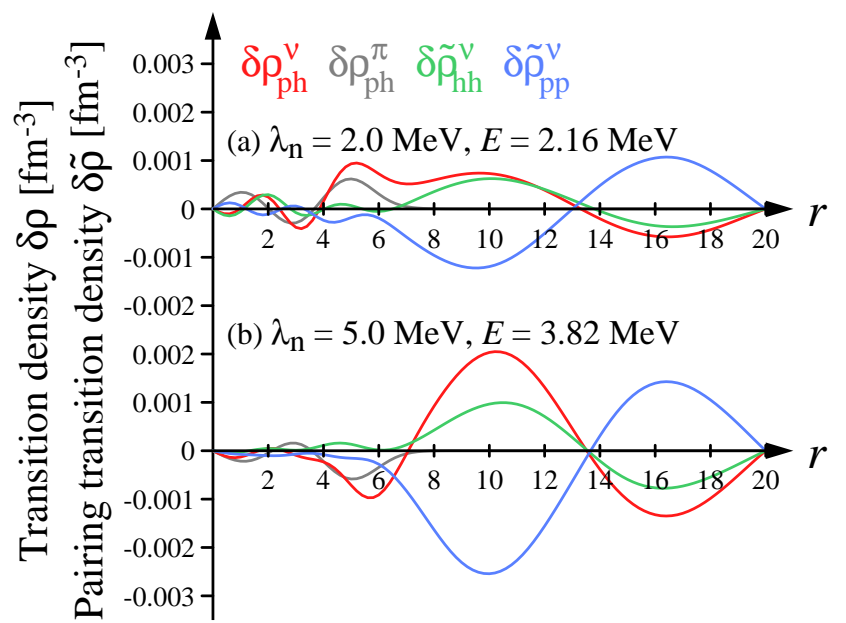

FIG. 11. (Color online) Transition densities $\delta \rho_{\mathrm{ph}}^{\nu}, \delta \rho_{\mathrm{ph}}^{\pi}, \delta \tilde{\rho}_{\mathrm{pp}}^{\nu}$, and $\delta \tilde{\rho}_{\text {hh }}^{\nu}$ of the second AB phonon state: (a) $2.16 \mathrm{MeV}$ state for $Z=28$ with $\lambda_{n}=2.0 \mathrm{MeV}$ and (b) $3.82 \mathrm{MeV}$ state for $Z=28$ with $\lambda_{n}=5.0 \mathrm{MeV}$.

ond excited state, a very small peak at $E=2.16 \mathrm{MeV}$ in Fig. [3, has partly the character of the second harmonics of the $\mathrm{AB}$ phonon mode. The transition densities of this state is shown in Fig. 11(a), which has an oscillation node at $r_{\text {node }} \approx 13 \mathrm{fm}$. (The small transition strengths are due to cancellation between the amplitudes in two regions $r>r_{\text {node }}$ and $r<r_{\text {node. }}$ ) However, the oscillatory behaviour is not as clear as the first $\mathrm{AB}$ phonon mode at $E=1.71 \mathrm{MeV}$. We deduce that the collectivity does not develop well as the excitation energy is comparable to $2 \Delta_{\text {ext }}$. Also for $\lambda_{n} \geq 3.0 \mathrm{MeV}$, we find a state interpreted as the second harmonics of the $\mathrm{AB}$ phonon mode. The excitation energies are $E=2.81,3.47,3.82$, $4.10 \mathrm{MeV}$ for $\lambda_{n}=3.0,4.0,5.0,6.0 \mathrm{MeV}$. One example is shown in Fig. 11(b). We see a clearer oscillatory profile in these cases, and it may be due to more developed collectivity, reflecting the excitation energy smaller than $2 \Delta_{\text {ext }}$.

\section{CONCLUSION}

We have investigated the Anderson-Bogoliubov (AB) phonon (called also superfluid phonon) appearing in the inner crust of neutron stars by employing the density functional description of nucleon many-body collective dynamics. We consider configurations where spherical clusters are immersed in neutron superfluid, and formulate the HFB plus QRPA model in a spherical WignerSeitz cell. We adopt the Skyrme energy density functional SLy4 and the density-dependent delta interaction, which are designed to describe not only finite nuclei but also the EOS and the pairing properties of neutron matter. In the present paper, we have focused on the $\mathrm{AB}$ phonon mode with the dipole multipolarity, relevant 
to the coupling to the lattice phonon or the displacement motion of clusters, and studied how the presence of clusters influences the AB phonon. Numerical analysis is performed by varying the neutron chemical potential $\lambda_{n}=1-6 \mathrm{MeV}$ (changing the density of superfluid neutrons) and the proton numbers of clusters.

Our model demonstrates systematic emergence of very collective low-energy excitations which display clearly typical characteristics of the AB phonon in the external region of clusters, i.e. in surrounding neutron superfluid. However, this AB phonon mode is modified strongly by the presence of clusters. The phonon amplitude is significantly reduced inside the surface of cluster as if the $\mathrm{AB}$ phonon does not penetrate into clusters. This suggests that the coupling of the $\mathrm{AB}$ phonon to the lattice phonon may be weaker than what is expected from a simple hydrodynamic description assuming uniform neutron superfluid. Examining in detail results from various neutron chemical potentials $\lambda_{n}$, we find also that behaviour of the AB phonon around the clusters depends on $\lambda_{n}$ or the density of surrounding neutron superfluid. At higher densities corresponding to $\lambda_{n} \gtrsim 4-5 \mathrm{MeV}$, the phonon amplitudes display a clear node in the surface area of clusters, as if the AB phonon in neutron superfluid gives recoil motion to core part of the clusters in opposite direction to the AB phonon outside. This behaviour resembles that of the pygmy dipole excitation of neutron-rich nuclei. This points to a possibility that the coupling between the lattice phonon and the AB phonon has non-trivial dependence on $\lambda_{n}$, and may become even weaker for $\lambda_{n} \gtrsim 4-5 \mathrm{MeV}$. Quantitative analysis of the phonon-phonon coupling and its impact on the thermal conductivity of inner crust will be discussed in forthcoming papers.

\section{ACKNOWLEDGMENTS}

This work is financially supported by Grant-in-Aid for Scientific Research on Innovative Areas, No. 24105008, by The Ministry of Education, Culture, Sports, Science and Technology, Japan.
[1] N. Chamel, and P. Haensel, Living Rev. Relativity, 11, 10 (2008).

[2] P. Haensel, A. Y. Potekhin, D. G. Yakovlev, Neutron Stars 1: Equation of State and Structure, Astrophysics and Space Science Library, vol.326 (Springer, New York, 2007).

[3] C. J. Pethick, D. G. Ravenhall, Annu. Rev. Nucl. Part. Sci. 45, 429 (1995).

[4] P. W. Anderson, N. Itoh, Nature, 256, (1975).

[5] M. Ali Alpar, Astrophys. J. 213, 527 (1977)

[6] D. Pines and M. Ali Alpar, in The Structure and Evolution of Neutron Stars, ed. D. Pines, R. Tamagaki, and S. Tsuruta ( Addison Wesley, 1992), p.7.

[7] J. M. Lattimer, K. A. van Riper, M. Prakash, and M. Prakash, Astrophys. J. 425, 802 (1994).

[8] O. Y. Gnedin, D. G. Yakovlev, and A. Y. Potekhin, Mon. Not. R. Astron. Soc. 324, 725 (2001).

[9] P. S. Shternin, D. G. Yakovlev, P. Haensel, and A. Y. Potekhin, Mon. Not. R. Astron. Soc. Lett. 382, L43 (2007).

[10] E. F. Brown and A. Cumming, Astrophys. J. 698, 1020 (2009).

[11] P. M. Pizzochero, F. Barranco, E. Vigezzi and R. A. Broglia, Astrophys. J. 569, 381 (2002).

[12] C. Monrozeau, J. Margueron, and N. Sandulescu, Phys. Rev. C 75, 065807 (2007).

[13] M. Fortin, F. Grill, J. Margueron, D. Page, and N. Sandulescu, Phys. Rev. C 82, 065804 (2010).

[14] R. C. Duncan, Astrophys. J. 498, L45 (1998).

[15] L. Samuelson, and N. Andersson, Mon. Not. R. Astron. Soc., 374, 256 (2007).

[16] N. Andersson, K. Glampedakis, and L. Samuelsson, Mon. Not. R. Astron. Soc. 396, 894 (2009).

[17] D. N. Aguilera, V. Cirigliano, J. A. Pons, S. Reddy, and R. Sharma, Phys. Rev. Lett. 102, 091101 (2009).
[18] C. J. Pethick, N. Chamel, and S. Reddy, Prog. Theor. Phys. Supple. 186, 9 (2010).

[19] V. Cirigliano, S. Reddy, and R. Sharma, Phys. Rev. C 84, 045809 (2011).

[20] D. Page and S. Reddy, in Neutron Star Crust, ed. by C. Bertulani and J. Piekarewicz (Nova Science, 2012), p. 281

[21] N. Chamel, D. Page, and S. Reddy, Phys. Rev. C 87, 035803 (2013); J. Phys, Conf. Ser., 665, 012065 (2016).

[22] D. Kobyakov and C. J. Pethick, Phys. Rev. C 87, 055803 (2013)

[23] D. Kobyakov and C. J. Pethick, Phys. Rev. Lett. 112, 112504 (2014).

[24] N. Martin and M. Urban, Phys. Rev. C 90, 065805 (2014).

[25] P. W. Anderson, Phys. Rev. 112, 1900 (1958).

[26] N. N. Bogoliubov, V. V. Tolmachev, and D. V. Shirkov, A New Method in the Theory of Superconductivity (Academy of Science, Moscow, 1958, New York, 1959).

[27] V. M. Galitskii, JETP 34, 1011 (1958).

[28] N. Chamel, S. Goriely, J. M. Pearson, and M. Onsi, Phys. Rev. C 81, 045804 (2010)

[29] N. Sandulescu, N. V. Giai, and R.J. Liotta, Phys. Rev. C 69, 045802 (2004).

[30] N. Sandulescu, Phys. Rev. C 70, 025801 (2004).

[31] M. Baldo, U. Lombardo, E. E. Sperstein, and S. V. Tolokonnikov, Nucl. Phys. A750, 409 (2005).

[32] M. Baldo, E. E. Sperstein, and S. V. Tolokonnikov, Nucl. Phys. A775, 235 (2006).

[33] F. Grill, J. Margueron, and N. Sandulescu, Phys. Rev. C 84, 065801 (2011).

[34] A. Pastore, S. Baroni, and C. Losa, Phys. Rev. C 84, 065807 (2011)

[35] A. Pastore, Phys. Rev. C 86, 065802 (2012). 
[36] F. Barranco, R. A. Broglia, H. Esbensen, and E. Vigezzi, Phys. Rev. C 58, 1257.

[37] E. Khan, N. Sandulescu, and N. V. Giai, Phys. Rev. C 71, 042801 (R) (2005).

[38] M. Grasso, E. Khan, J. Margueron, N. V. Giai, Nucl. Phys. A 807, 1 (2008).

[39] G. Gori, F. Ramponi, F. Barranco, R. A. Broglia, G. L. Colo, D. Sarchi, Nucl. Phys. A731, 401 (2004).

[40] S. Baroni, A. Pastore, F. Raimondi, F. Barranco, R. A. Broglia, and E. Vigezzi, Phys. Rev. C 82, 015807 (2010).

[41] T. Nakatsukasa, K. Matsuyanagi, M. Matsuo, and K. Yabana, Rev. Mod. Phys. 88, 045004 (2016).

[42] M. Matsuo, Nucl. Phys. A 696, 371 (2001); Prog. Theor. Phys. Suppl. 146, 110 (2002).

[43] M. Matsuo, K. Mizuyama, and Y. Serizawa, Phys. Rev. C 71, 064326 (2005).

[44] Y. Serizawa and M. Matsuo, Prog. Theor. Phys. 121, 97 (2009).

[45] M. Matsuo and Y. Serizawa, Phys. Rev. C 82, 024318 (2010).
[46] J. W. Negele and D. Vautherin, Nucl. Phys. A 207, 298 (1973).

[47] E. Chabanat, P. Bonche, P. Heenen, J. Meyer, and R. Schaeffer, Nucl. Phys. A 635, 231 (1998).

[48] A. Akmal, V. R. Pandharipande, and D. G. Ravenhall, Phys. Rev. C 58, 1804 (1998).

[49] M. Matsuo, Y. Serizawa, and K. Mizuyama, Nucl. Phys. A 788,307c (2007).

[50] M. Matsuo, Phys. Rev. C 73, 044309 (2006).

[51] E. Khan, N. Sandulescu, N. V. Giai, and M. Grasso, Phys. Rev. C 69, 014314 (2004).

[52] E. Khan, M. Grasso, and J. Margueron, Phys. Rev. C 80, 044328 (2009).

[53] N. Paar, D. Vretenar, E. Khan, and G. Colò, Rep. Prog. Phys. 70, 691 (2007).

[54] P. Papakonstantinou, J. Margueron, F. Gulminelli, and Ad. R. Raduta, Phys. Rev. C 88, 045805 (2013).

[55] C. J. Pethick and H. Smith, Bose-Einstein Condensation in Dilute Gases, (Cambridge University Press, Cambridge, 2002). 